\title{
Estrategias de aprendizaje de la gestión de riesgos a partir de los estilos de aprendizaje de estudiantes de la Facultad de Recursos Naturales y del Ambiente - UNA
}

\section{Risk management learning strategies based on the learning styles of students in the Facultad de Recursos Naturales y del Ambiente - UNA}

\section{César Aguirre Jiménez}

Ingeniero Agrónomo, ORCID: https://orcid.org/0000-0002-9363-6627 / caguirre@ci.una.edu.ni Facultad de Recursos Naturales y del Ambiente, Universidad Nacional Agraria (UNA)

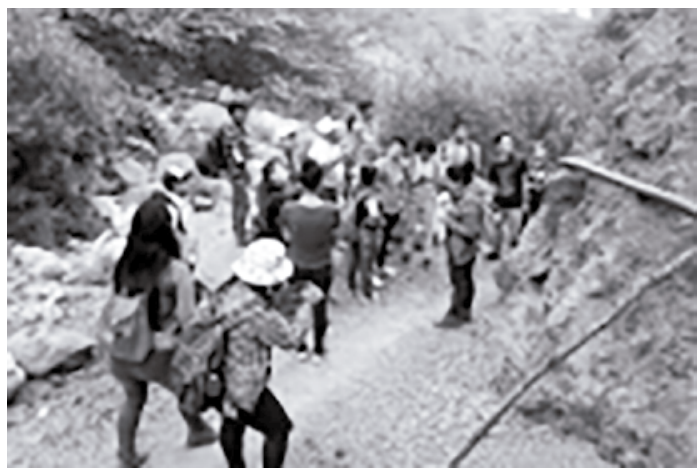

\section{RESUMEN}

El estudio se desarrolló con estudiantes de la Facultad de Recursos Naturales y del Ambiente (FARENA) - Universidad Nacional Agraria (UNA), bajo el supuesto que el conocimiento de los estilos de aprendizaje (EA) de la comunidad estudiantil permite identificar estrategias para la mejora del aprendizaje de la gestión de riesgos. La metodología incluyó el análisis del contexto y el diagnóstico de los estilos de aprendizaje de estudiantes de FARENA, utilizando el Cuestionario Honey - Alonso de Estilos de Aprendizaje. Los resultados indican que las y los estudiantes tienden a utilizar los EA pragmático y teórico en una escala alto, y el reflexivo y el activo en una escala moderado; no obstante, se encontraron diferencias significativas por edad, carrera y año académico. Los hallazgos dejan en evidencia que existe oportunidad de mejora del aprendizaje de la Gestión de Riesgo (GdR), mediante estrategias de enseñanza aprendizaje que tomen en consideracion los estilos de aprendizaje de la comunidad estudiantil. Al profesorado le corresponde estructurar situaciones de aprendizaje de la GdR, que sean coherentes con los estilos preferentes de aprender de varones y mujeres de cada una de las Carreras de FARENA.

Palabras clave: riesgo de desastres, estrategias de enseñanza - aprendizaje, universidad.

\section{ABSTRACT}

The study was developed with students from the Facultad de Recursos Naturales y del Ambiente (FARENA) - Universidad Nacional Agraria (UNA), under the assumption that knowledge of the learning styles (LS) of the student community allows to identify strategies for improving risk management learning. The methodology included context analysis and diagnosis of FARENA student learning styles, using the Honey - Alonso Learning Styles Questionnaire. The results indicate that students tend to use pragmatic and theoretical LS on a high scale, and the reflective and active on a moderate scale; however, significant differences were found by age, career and academic year. The findings show that there is an opportunity to improve the learning of the Risk Management (RM), through teaching strategies learning that take into consideration the learning styles of the student community. It is up to teachers to structure Learning Situations of the $\mathrm{RM}$, which are consistent with the preferred styles of learning of men and women from each of the FARENA Careers.

Keywords: Learning styles, risk management, students, university.
Recibido: 12 de diciembre del 2018 Aceptado: 8 de julio del 2019

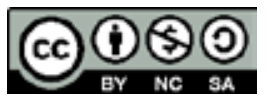

Los artículos de la revista La Calera de la Universidad Nacional Agraria, Nicaragua, se comparten bajo términos de la licencia Creative Commons: Reconocimiento, No Comercial, Compartir Igual. Las autorizaciones adicionales a las aquí delimitadas se pueden obtener en el correo edgardo.jimenez@ci.una.edu.ni 
$\mathrm{A}$

nte los grandes problemas sociales, económicos y ambientales que enfrenta la humanidad, tales como la degradación ambiental, la desigualdad y el consecuente aumento de los desastres, la educación se ha erigido como el instrumento que hace resurgir en las sociedades la esperanza de lograr los cambios requeridos para evitar los desastres o disminuir los efectos de la ocurrencia de eventos adversos. De ahí la necesidad de una educación en GdR, como un factor fundamental para reducir vulnerabilidades y desarrollar capacidades para afrontar emergencias y desastres. En este contexto y en correspondencia con los cambios que experimenta la educación superior a nivel internacional, en el año 2011 la Universidad Nacional Agraria (UNA) aprobó un nuevo Modelo Educativo con un enfoque pedagógico socio-constructivista ecológico y un diseño curricular basado en competencias. Sin embargo, se identifican debilidades en los procesos de enseñanza - aprendizaje; según el Informe de Autoevaluación 2013-2015 (UNA, 2015), al respecto del grado de satisfacción de la metodología de enseñanza - aprendizaje "en promedio el $69 \%$ de los estudiantes y el $91 \%$ de docentes mostraron satisfacción por la metodología". Estos datos señalan la necesidad de mayor información respecto a las preferencias en el aprendizaje del estudiantado.

Por otro lado, como parte del proceso de transformación curricular se establecieron los tópicos Adaptación al Cambio Climático (ACC) y la Gestión de Riesgos ante Desastres (GdRD) como ejes transversales en la nueva oferta académica. Esto supone un doble desafío: la identificación de oportunidades de aprendizaje de la GdR en cada uno de los módulos de las carreras que ofrece la UNA, y el diseño de acciones metodológicas para que los futuros profesionales desarrollen las competencias necesarias que contribuyan a un desarrollo humano, seguro y sostenible. La educación en GdR es un proceso educativo complejo que implica el abordaje de las dimensiones biofísicas, sociales, económicas y político - administrativas, propias de la dinámica territorial; por tanto, se requiere del diseño de estrategias de enseñanza - aprendizaje que faciliten el aprendizaje. Es un proceso formativo que promueve la solidaridad, los valores, el compromiso ético y moral con la vida y el ambiente.

Según Ramírez (2011), es una educación dirigida a conocer los riesgos a que están expuestas las sociedades y el ambiente, y las medidas para reducirlos y mitigarlos, así como a responder a los eventos adversos y a recuperarse; a hacer las cosas de manera que no lleguen a crear más riesgos de los existentes sino que contribuyan a reducirlos; a ser solidarios en momentos de calamidad; a ser respetuosos con la vida y con la seguridad de sus semejantes; a ser íntegros, responsables, comprometidos con la vida en todas sus formas; a aprender a emprender, a cambiar, a innovar para reducir el riesgo de desastres y adaptarse al cambio climático.

Por tanto, se trata de un proceso educativo caracterizado por ser complejo (porque implica el abordaje de fenómenos relacionados con las dimensiones biofísicas, sociales, económicas y político - administrativas en un territorio); sistémico (en el sentido que involucra todo el sistema educativo, así como en el análisis de causas y las consecuencias); interdisciplinar (requiere de la intervención de diversas disciplinas de las ciencias naturales, físicas y sociales, de manera interrelacionada); integral (se orienta a lo cognoscitivo, ético y moral de los sujetos y las poblaciones, para lograr estilos de vida comprometidos con la prevención de desastres y el desarrollo sustentable); e integrador (ya que requiere de la intervención y el esfuerzo de los diferentes actores vinculados con la comunidad educativa).

No obstante, no significa que se trate de la instauración de una educación exclusiva para la reducción de riesgos de desastres, sino de fortalecer la educación integral como un factor fundamental para reducir vulnerabilidades y desarrollar capacidades para afrontar emergencias y desastres. Según Alpízar (2009) hay una serie de aspectos a fortalecer para reducir desastres desde el sector educativo, los cuales pueden agruparse en dos grandes componentes: a) la gestión prospectiva del riesgo, que incluye acciones dirigidas a evitar la generación de nuevas vulnerabilidades en distintos contextos, de forma prospectiva y anticipada; y b) desde la preparación y atención ante emergencias y desastres, abarca los planes de reducción de vulnerabilidad y atención de emergencias, la capacitación permanente en las formas de actuación en situaciones de emergencia.

En este sentido, el uso del modelo de aprendizaje experiencial de David Kolb (1984) en los procesos de enseñanza - aprendizaje de la GdR en la Universidad, se justifica por ser uno de los más conocidos y aplicados en el desarrollo de competencias. Hay suficiente evidencia que respalda que el diseño de acciones metodológicas para la educación a partir de los estilos de aprendizaje preferentes del estudiantado, puede brindar las herramientas necesarias para incitar el aprendizaje de la GdR y lograr la metacognición que permita el entendimiento de fenómenos socio-culturales y el desarrollo de habilidades complejas para gestionar el riesgo de desastres; asimismo, está acorde con el Modelo Educativo de la UNA. 
Los estilos de aprendizaje pueden ser de mucha ayuda para la comunidad docente en el proceso de planificación de acciones metodológicas; éstos hacen referencia a los procesos de adquirir conocimientos, lo cual implica las acciones y emociones para obtener, procesar, comprender y poner en práctica una información. Tal como plantean Alonso y Gallego (2004, p. 8), "tres elementos psicológicos primarios conforman el estilo: un componente afectivo, el sentimiento, un componente cognitivo, el conocer, un componente de comportamiento, el hacer. Estos elementos se estructuran según el propio estilo y reflejan el modo por el que la persona construye su proceso de aprendizaje".

En el contexto actual en que el estudiantado espera un ambiente centrado en su aprendizaje, que les permita interactuar y participar de acuerdo a sus intereses, se demanda experiencias que estén más acordes a sus estilos preferentes de aprender. Tal como indica Ventura (2011, p.149), "las investigaciones empíricas de los últimos diez años comparten el postulado de que los estilos de aprendizaje se constituyen como herramientas de apoyo psicopedagógico. Estas tendencias impulsan propuestas educativas adecuadas a los procesos de aprendizaje, entendiendo a la atención de la diversidad como una de sus condiciones fundantes... Se considera que cuando el docente diseña estrategias de enseñanza de acuerdo a los estilos preferenciales de sus estudiantes, se facilita el proceso de aprendizaje

\section{METODOLOGÍA}

La perspectiva de investigación empleada es de tipo exploratoria-descriptiva, por medio de la cual se caracterizó los estilos de aprendizaje utilizados por el estudiantado de FARENA-UNA. El instrumento utilizado fue el Cuestionario Honey - Alonso de Estilos de Aprendizaje (CHAEA), debido a que es uno de los más empleado en estudios similares en universidades iberoamericanas. Participaron en el estudio 310 estudiantes voluntarios, correspondiente al $64 \%$ del total de estudiantes que en 2015 cursaban las carreras Ingeniería en Recursos Naturales Renovables (IRNR) e Ingeniería Forestal.

Una vez recopilados los cuestionarios CHAEA, se procedió a la elaboración de base de datos, para su posterior procesamiento y análisis estadístico. La puntuación $(+o-)$ de cada cuestionario se transcribió en una hoja de cálculo del Programa Excel. Para identificar las tendencias del comportamiento de uso de los estilos de aprendizaje, se utilizó la estadística descriptiva; el programa Infostaf 2009 se utilizó en el análisis de la media y la varianza de los datos. Los gráficos se generaron mediante los pro- gramas Excel y Sigmaplot 12, lo cual permitió reflejar los resultados de manera visual y más resumida. Los resultados se presentan en tablas de salida y graficas de barras.

\section{RESULTADOS Y DISCUSIÓN}

Estilos de aprendizaje de la población estudiantil de la FARENA. La población estudiantil de la FARENA participante en el estudio fue de 310 estudiantes: 106 estudiantes pertenecientes a IRNR, de los cuales 45 eran mujeres (42 $\%$ ) y 61 hombres (58\%); en el caso de IF participaron 204 estudiantes, 73 mujeres (36\%) y 131 hombres (64\%).

El análisis global de los resultados indica que las y los estudiantes de FARENA tienden a utilizar los estilos de aprendizaje pragmático y teórico en una escala alto, y el activo y el reflexivo en una escala moderado (ver figura 1); no obstante, el análisis estadístico muestra diferencias significativas por edad, carrera, y año académico. En el caso del uso del estilo activo se observa diferencia por sexo, ya que los hombres lo usan en una escala alto y las mujeres en la escala moderado; no obstante, sólo hay diferencias significativas en el estilo teórico.

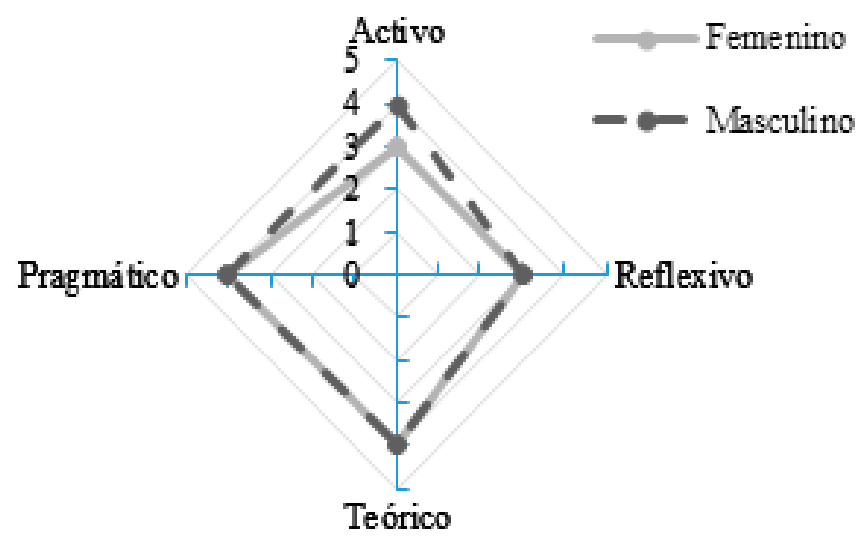

Figura 1. Escalas de uso de los estilos de aprendizaje de estudiantes de FARENA.

Escalas de uso: 1, muy bajo; 2, bajo; 3, moderado; 4, alto; 5, muy alto. Sexo: M, masculino; F, femenino. Fuente: elaborado a partir de los CHAEA de estudiantes de FARENA.

Estos resultados difieren en parte de lo reportado por Alonso, Gallego y Honey (s.f.), quienes indican que los estudiantes de Facultades Experimentales de España usan en primera posicion los estilos teórico y reflexivo, y en menor medida los estilos activo y pragmático. Sin embargo, se concuerda con Villalba (2015) respecto a que hay estudios que divergen de ese perfil de estilos de aprendizaje; en el caso de estudiantes argentinos de las carreras de Biología y Biodiversidad encontró los perfiles de esti- 
los de aprendizaje Reflexivo-Activo-Pragmático-Teórico al ingresar y Reflexivo-Teórico-Activo-Pragmático próximos a egresar.

Estilos de aprendizaje de estudiantes de IF. Tal como puede observarse en la figura 2, la mediana indica que hay diferencias en la preferencia de uso de los estilos de aprendizaje por año académico: el estudiantado de I y II Año tiende a usar los estilos activo, teórico y pragmático en la escala alto, y el reflexivo en escala moderada; el de III y IV Año usa los cuatro estilos en escala moderada; el de V Año usa los estilos activo y teórico en escala alto, y los estilos reflexivo y pragmático en la escala moderada.

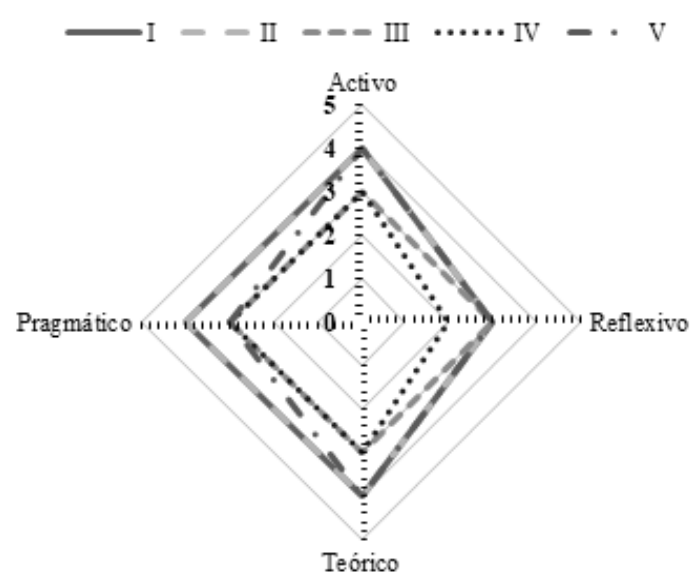

Figura 2. Escalas de uso de los estilos de aprendizaje de estudiantes de IF por año académico.

Escalas de uso: 1, muy bajo; 2, bajo; 3, moderado; 4, alto; 5, muy alto. Fuente: elaborado a partir de los CHAEA de estudiantes de IF.

Mayor diferencia se observa al considerar sólo la proporción de estudiantes por sexo, que usan los estilos en las escalas alto y muy alto; en el caso de los hombres el perfil de los estilos de aprendizaje es teórico (59\%), pragmático (56\%), activo (55\%), y en menor proporción el reflexivo (24\%). En cambio, en las mujeres es activo (50 $\%)$, teórico (41\%), y en menor proporción el pragmático (33\%) y reflexivo $(19 \%)$.

Estilos de aprendizaje de estudiantes de IRNR. A diferencia de lo observado en la carrera de IF, en IRNR hay un mayor porcentaje de estudiantes que usan los estilos de aprendizaje en la escala alto; en I, III y IV Año usan los estilos activo, teórico y pragmático en escala alto y el reflexivo en escala moderada. Mientras que en II y V Años usan sólo el estilo pragmático en escala alto y los estilos activo, reflexivo y teórico en escala moderada (Figura 3).

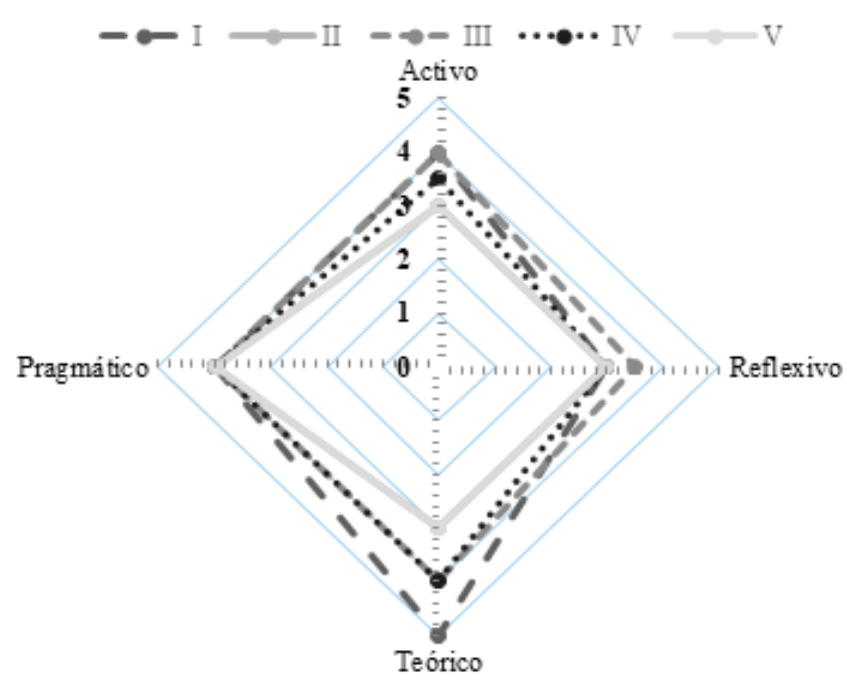

Figura 3. Escalas de uso de los estilos de aprendizaje de estudiantes de IRNR por año académico.

Escalas de uso: 1, muy bajo; 2, bajo; 3, moderado; 4, alto; 5, muy alto. Fuente: elaborado a partir de los CHAEA de estudiantes de IRNR.

Al igual que IF, se observa mayor diferencia al considerar sólo la proporción de estudiantes por sexo que usan el estilo en las escalas alto y muy alto; en el caso de los hombres el perfil de los estilos de aprendizaje es pragmático (62\%), teórico (52\%), activo (48 \%) y en menor proporción el reflexivo (35\%). En el caso de las mujeres el perfil es teórico $(75 \%)$, pragmático $(62 \%)$, activo (51 $\%)$ y en menor medida el reflexivo (22\%).

Como puede apreciarse, a la mayoría de estudiantes les cuesta más ser reflexivos, cautelosos, trabajar a conciencia, interpretar bien antes de emitir su opinión.

\section{Cualidades que caracterizan los estilos de aprendiza-} je. A partir de las preferencias marcadas (mayores al 70 \%) en el CHAEA por las y los estudiantes de FARENA, los rasgos principales que describen sus estilos de aprendizaje son los siguientes:

Estilo activo. Son personas espontáneas, les gusta asumir nuevos retos, abiertos, gozan el momento, prefieren ideas originales y novedosas.

Estilo reflexivo. son personas concienzudas, observadoras, pausadas, reflexivas, cautelosas, tratan de interpretar bien antes de emitir su opinión.

Estilo teórico. son equilibradas, coherentes, estructuradas, serias, lógicas, buscan conclusiones e ideas claras. 
Estilo pragmático. Son prácticas, realistas, experimentadoras, directas, les gusta que las cosas funcionen, apoyan ideas prácticas y realistas.

Estrategias de aprendizaje de la GdR a partir de los estilos de aprendizaje. Los perfiles de estilos de aprendizaje de la población estudiantil de FARENA reflejan que hay una proporción importante de estudiantes que requieren mejorar sus estrategias para obtener, procesar, comprender y poner en práctica una información, de manera que puedan convertirse en aprendices estratégicos. Tal como plantea Alonso y Gallego (2010), los estilos de aprendizaje podrían desarrollarse como competencias necesarias para el estudio, el trabajo y la vida: buscar información (activo), ante la mayor cantidad y de fuentes diferentes; saber analizarla (reflexivo), para estudiar y profundizar los diferentes matices de la información; estructurarla (teórico), para sintetizarla e integrarla a la existente; aplicarla (pragmático), para ponerla en práctica, construir conocimiento.

Los resultados indican que hay estudiantes que usan en escala alto dos o tres estilos de aprendizaje, pero también hay una proporción importante que los usa en escala moderada y algunos en escala baja; por tanto, hay oportunidad para que puedan utilizar sus puntos fuertes y trabajar para desarrollar los estilos que utilizan en esca- la baja y moderada, de manera que se pueda favorecer el aprendizaje de la GdR. Según Alonso, Gallego y Honey (s.f.), para fortalecer los estilos de aprendizaje, se debe establecer un plan de acción que incluya lo siguiente:

a. Identificar puntos debiles: luego de completar el CHAEA es necesario identificar los puntos debiles en cada estilo.

b. Decidir sobre los aspectos que les gustaría practicar para fortalecerlos (objetivos a lograr); se recomienda no seleccionar mas de tres aspectos marcados + o - (o combinacion de ambos), por cada estilo de aprendizaje. Es conveniente redactarlos nuevamente en sus propias palabras.

El diagnóstico presentado deja en evidencia que existe oportunidad de mejora pedagógica para un aprendizaje efectivo de la GdR, definiendo estrategias de enseñanza - aprendizaje que sean coherentes con los estilos de aprendizaje de la comunidad estudiantil. Al profesorado le corresponde estructurar situaciones de aprendizaje de la GdR, que se adapten a los estilos preferentes de aprender de varones y mujeres, de cada una de las Carreras de FARENA. En el cuadro 1 se muestran sugerencias de estrategias de aprendizaje adaptadas de Alonso, Gallego y Honey (s.f.), para fortalecer la educación en GdR a partir de los cuatro estilos de aprendizaje.

Cuadro 1. Sugerencias para fortalecer la educación en GdR a partir de los estilos de aprendizaje

\begin{tabular}{|c|c|c|c|}
\hline Estilo Activo & Estilo Reflexivo & Estilo Teórico & Estilo Pragmático \\
\hline $\begin{array}{l}\text { - Hacer actividades nuevas para } \\
\text { el aprendizaje de la GdR al } \\
\text { menos una vez por semana. } \\
\text { - Practicar diálogos con estu- } \\
\text { diantes de otras carreras para } \\
\text { convencer sobre la importan- } \\
\text { cia de la educación en GdR. } \\
\text { - Cambiar de actividades de re- } \\
\text { flexión a prácticas cada media } \\
\text { hora. } \\
\text { - Forzarse a ejercer liderazgo } \\
\text { en prácticas o simulacros de } \\
\text { situaciones de emergencias. } \\
\text { - Investigar, formular proble- } \\
\text { mas relacionados con la GdR. }\end{array}$ & $\begin{array}{l}\text { - Observar situaciones de vulne- } \\
\text { rabilidad, estudiar el comporta- } \\
\text { miento en esas condiciones. } \\
\text { - Llevar diario personal de re- } \\
\text { flexiones sobre la GdR. } \\
\text { - Revisar lo ocurrido en situaciones } \\
\text { de emergencias y sacar conclu- } \\
\text { siones, lecciones aprendidas. } \\
\text { - Prevenir a personas activas para } \\
\text { analicen consecuencias y consi- } \\
\text { deren alternativas de RRD -ACC. } \\
\text { - Investigar temas de GdR en dife- } \\
\text { rentes fuentes. } \\
\text { - Escribir ensayos, informes, artí- } \\
\text { culos sobre la GdR. } \\
\text { - Revisar los escritos luego de una } \\
\text { semana y mejorarlos. } \\
\text { Practicar listas a favor y en contra } \\
\text { en temas sobre la GdR. }\end{array}$ & $\begin{array}{l}\text { - Leer a diario por } 30 \text { minutos material } \\
\text { sobre GdR y hacer resumen con sus pro- } \\
\text { pias palabras. } \\
\text { - Detectar incoherencias o puntos débiles } \\
\text { en argumentos de otras personas sobre } \\
\text { CC. } \\
\text { - Analizar casos de actuaciones en situa- } \\
\text { ciones de desastres y señalar por qué se } \\
\text { hizo de esa manera, qué se pudo hacer de } \\
\text { otra forma y cómo. } \\
\text { - Resumir teorías/ hipótesis sobre causas y } \\
\text { consecuencias de los desastres. } \\
\text { Estructurar medidas de adaptación/miti- } \\
\text { gación para la RRD - ACC. } \\
\text { Inventar métodos / procedimientos para } \\
\text { la RRD - ACC. } \\
\text { Hacer preguntas exigentes y solicitar res- } \\
\text { puestas concretas sobre la RRD - ACC. }\end{array}$ & $\begin{array}{l}\text { - Reunir técnicas, modos prácti- } \\
\text { cos de RRD-ACC. } \\
\text { - Recabar ayuda de personas ex- } \\
\text { perimentadas. } \\
\text { - Elaborar planes de acción para } \\
\text { la RRD - ACC. } \\
\text { - Practicar técnicas novedosas } \\
\text { para la ACC. } \\
\text { Evitar implementar medida de } \\
\text { RRD-ACC con riesgo de fraca- } \\
\text { so elevado. } \\
\text { Estudiar técnicas de RRD - } \\
\text { ACC y luego tratar de adaptar- } \\
\text { las a un contexto. } \\
\text { Emprender un proyecto de RRD } \\
\text { - ACC en escuelas o comunida- } \\
\text { des. }\end{array}$ \\
\hline
\end{tabular}

Fuente: Adaptado de Alonso, Gallego y Honey (s.f.). 
Por otro lado, se reconoce que los estilos de aprendizaje del profesorado influyen en sus estilos de enseñanza, por tanto, es deseable que se hagan esfuerzos para perfeccionarlos. En la medida que las y los docentes dominen con propiedad los cuatro estilos, serán capaces de enseñar a la población estudiantil el cómo potenciar sus estilos de aprendizaje, mejorando así los procesos de enseñanza - aprendizaje de la GdR. Especial importancia se debe dar al fortalecimiento del estilo reflexivo, ya que es fundamental para desarrollar la competencia de aprender a aprender.

Asimismo, es necesario resaltar que los desastres afectan de manera diferenciada a hombres y mujeres; las relaciones de género tienden a desempeñar un rol importante en la estructuración del contexto y, por tanto, son trascendentales para la construcción de la vulnerabilidad y el riesgo, y sus efectos. Solo si se aplica un enfoque de género es posible adquirir una idea más clara y completa de las relaciones que han desarrollado las personas con los ecosistemas. Hombres y mujeres enfrentan su realidad social, económica y ambiental de manera diferente; por tanto, la manera en que participan también es diferente y tiene una estrecha relación con la edad, nivel socioeconómico y cultural.

Por consiguiente, el profesorado debe abordar la educación en GdR desde el enfoque de género, para que el estudiantado comprenda de qué manera las identidades de mujeres y hombres determinan diferentes vulnerabilidades y capacidades, pero también como herramienta para el análisis de pertinencia y efectividad de políticas y acciones de RRD - ACC, que se implementan desde el nivel internacional hasta el local.

\section{CONCLUSIONES}

El conocimiento de los estilos de aprendizaje por parte de estudiantes y docentes de la FARENA brinda la oportunidad de saber las formas preferentes de aprender, y a partir de ello derivar acciones metodológicas que faciliten el aprendizaje de la GdR, asi como un mejor desenvolvimiento en el ámbito complejo y de múltiples cambios de la época actual.

El diagnóstico de los estilos de aprendizaje de estudiantes de FARENA, utilizando el CHAE, muestra que el perfil de estilos de aprendizaje es pragmático y teórico en una escala alto, y activo y reflexivo en escala moderado. Se observa que utilizan procesos y estrategias particulares para obtener, procesar, comprender y poner en práctica una información; asimismo, hay estudiantes que usan en escala alto dos o tres estilos de aprendizaje, pero la mayor proporción los usa en escala moderado y algunos en escala bajo. Por tanto, se refleja la necesidad de incorporar diferentes estrategias pedagógicas que atiendan la diversidad en el aula.

La educacion en GdR demanda el dominio de nuevos conocimientos y enfoques sobre cómo aprenden las personas. El estudio indica que existe oportunidad de mejora pedagógica para el aprendizaje de la GdR, definiendo estrategias de enseñanza - aprendizaje pertinentes y eficaces, que tomen en consideracion los estilos de aprendizaje de la comunidad estudiantil. Al profesorado le corresponde estructurar situaciones de aprendizaje de la GdR con un enfoque género, que se adapten a los estilos preferentes de aprender de varones y mujeres de cada una de las Carreras de FARENA.

\section{REFERENCIAS BIBLIOGRÁFICAS}

Alonso, C. y Gallego, D. (2010). Los estilos de aprendizaje como competencias para el estudio, el trabajo y la vida. Estilos de Aprendizaje, 6(6), 4-22.

Alonso, C., Gallego, D. y Honey, P. (s.f.). Los estilos de aprendizaje: procedimientos de diagnósticos y mejora. Mensajero. Bilbao, España. Bilbao: Mensajero.

Alpízar, M. (2009). Educación y Reducción de Riesgos y Desastres en Centroamérica: Gestión del Riesgo. San José: Coordinación Educativa y Cultural Centroamericana (CECC/SICA).

Kolb, D. (1984). Experiential learning: Experience as the source of learning and develoment. Englewood Cliffs, New Jersey: Prentice-Hall.

Ramírez, M. (2011). La educación en la gestión del riesgo de desastres. Una experiencia de todos y todas. En U. (Dir.), Educación y Gestión del Riesgo: Una experiencia para compartir. San José: Internem.

UNA (Universidad Nacional Agraria). (2015). Informe de Autoevaluación Institucional 2013 - 2015. Managua, Nicaragua.

Ventura, A. (2011). Estilos de aprendizaje y prácticas de enseñanza en la universidad: Un binomio que sustenta la calidad educativa. 142-154. Perfiles Educativos, 33(SPE), 142-154.

Villalba, A. (2015). Estilos de aprendizaje en alumnos universitarios de profesorado en biología y licenciatura en biodiversidad. Revista de Estilos de Aprendizaje, 8(16), 79-100. 